Full Length Article

\title{
The role of objects and effects in action imitation: Comparing the imitation of object-related actions vs. gestures in 18-month-old infants
}

\author{
Ziyon Kim ${ }^{\mathrm{a}, *}$, Gabriella Óturai ${ }^{\mathrm{a}}$, Ildikó Király ${ }^{\mathrm{b}}$, Monika Knopf ${ }^{\mathrm{a}}$ \\ a Department of Psychology, Goethe-University Frankfurt am Main, Theodor-W.-Adorno-Platz 6, Frankfurt am Main 60323, Germany \\ ${ }^{\mathrm{b}}$ Eötvös Loránd University, Budapest, Hungary
}

\section{A R T I C L E I N F O}

\section{Article history:}

Received 9 March 2015

Received in revised form 28 July 2015

Accepted 29 July 2015

\section{Keywords:}

Object-related actions

Gestures

Imitation

Action effects

Imitation ability

Social motivation

Cognitive motivation

\begin{abstract}
A B S T R A C T
This study aimed to systematically investigate 18-month-old infants' imitation of objectrelated actions compared to motorically similar gestures. An additional goal of the study was to examine the role of action effects on infants' imitation of target actions. One group of infants $(n=17)$ observed object-related actions and gestures leading to salient effects (sounds or visual resp. social effects), and the other group $(n=16)$ watched the same actions without effects. Furthermore, this study examined whether infants show a consistent imitation ability for object-related actions and gestures. First, the present study showed that 18-month-old infants imitated object-related actions more frequently than gestures. Second, the presence of an effect significantly increased the imitation rate of object-related actions; however, this difference was not found for gestures. Third, indications for a general imitation ability were found as results on an individual level showed that object-related action imitation significantly correlated with gesture imitation. Implications of the results for theory and future studies are discussed with a focus on the role of objects and effects in 18-month-old infants' action imitation.
\end{abstract}

(c) 2015 Elsevier Inc. All rights reserved.

\section{Introduction}

Imitation, which can be defined as performing an action after perceiving it (Bekkering, Wohlschläger, \& Gattis, 2000), is an important learning mechanism during infancy and childhood. Through the observation and repetition of others' actions, infants are able to learn one to two new behaviors each day (Barr \& Hayne, 2003). This observational learning enables infants to acquire many new skills in a reduced amount of time due to the skip of trial-and-error learning (Bekkering et al., 2000).

In a typical imitation task, a model performs novel actions on one or a series of objects in front of the infant. Therefore, this can be defined as a form of teaching situation, where the model shows an infant what an object is for or what can be done with it. However, acquiring new object-related skills is not the only reason that infants copy others. Since an imitation situation is also an interactive setting, social learning takes place within the interactive exchange between the model and the infant (Matheson, Moore, \& Akhtar, 2013).

Hence, imitation has two widely acknowledged functions: a cognitive function to learn something about the object or action and a social function to interact and affiliate with the model (Uzgiris, 1981).

\footnotetext{
* Corresponding author. Tel.: +49069798 35262 .

E-mail address: kim@psych.uni-frankfurt.de (Z. Kim).
} 
The function of imitation for the child at the moment of the demonstration is important in determining which aspect of a demonstration the child is likely to copy (Carpenter, 2006; Over \& Carpenter, 2012). Although the cognitive function and the social function co-occur, the cognitive function might become more apparent when infants are presented with novel object-related actions. In this form of imitation, infants focus much of their attention on the functions of the objects in order to learn a new skill and are accordingly mainly interested to attain a particular result (see also Zmyj, Aschersleben, Prinz, \& Daum, 2012). This is especially true in the case of younger infants as they focus more on the cognitive components in a demonstration situation. Infants around 12 months imitate primarily in order to satisfy cognitive motivations (e.g., Nielsen, 2006). However, with increasing age the social function of imitation becomes more important in general: older infants, from 18 months onward, imitate the specific actions of a model even if the actions are unsuccessful or irrational in order to promote interaction with the model and to satisfy social motivations. For example, in Nielsen's study (2006) infants saw a model's demonstration to use an object to open a box. The findings showed that 12-month-olds reproduced the model's results but did not copy the specific actions used by the model, unless they were given a rational reason to do so. Contrary to this, most of 18- and 24-month-olds imitated the model's object use, irrespective of the apparent logic of the actions. Further results showed that 18-month-olds copied the specific modeled action sequence with high fidelity when the model was being social while they focused on performing the end results when the model was acting aloof.

The conclusion that social motivations start to have a greater impact on infants' imitation at around 18 months of age (e.g., Carpenter, 2006; Nielsen, 2006) was mainly based on studies that were conducted with object-related actions. Given the general view that imitation is also a tool for learning within other non-object-related action types as well, the question arises whether the impact of social motivation to imitate varies in relation to the action type of use. When non-object-related actions such as gestures are given as a learning material in a demonstration situation, the target behavior ask for both cognitive and social motivation, yet as the cognitive task of understanding the observed action is related to a social partner (what is the model communicating/showing to me?), necessarily infants focus more on the social aspects of the imitation situation. Therefore, the above questions can be answered with the help of comparing the imitation of object-related actions to similar actions introduced as gestures.

So far only a few studies have compared infants' imitation of gestures to object-related actions (e.g., Abravanel, LevanGoldschmidt, \& Stevenson, 1976; Christie \& Slaughter, 2009; Rodgon \& Kurdek, 1977). These studies showed that infants are less likely to imitate gestures and that the imitation level is relatively low. For example, Abravanel et al. (1976) reported that 12- and 15-month-old infants imitated actions with objects more frequently than actions without objects, such as tongue protrusions, smacking lips and shaking of the head. Likewise, in the study of Christie and Slaughter (2009), six object-related actions (e.g., shaking a rattle) and nine bodily gestures (e.g., pulling the earlobe and patting the head) were shown to 6- and 15 -month-old infants. Only one of the 62 infants imitated a gestural action, whereas most of the infants imitated at least some of the object-related actions. Importantly, these studies compared object-related actions with gestures that differed in their motor components, thus only limited conclusions can be drawn. As infants have to draw on different motor or cognitive abilities in order to perform different actions, such study designs cannot exclude the impact of motor complexity in the imitation of different kinds of target actions. In addition, there has been no systematic investigation of older infants' imitation of object-related actions and comparable gestures.

Furthermore, compared to the amount of studies about object-related action imitation, the question whether gesture imitation is influenced by similar factors as object-related action imitation or not has received little attention. A substantial number of studies have reported that the presence of salient action effects (e.g., light or sound effect) has a strong influence on infants' imitation of object-related actions. Studies showed that action effects, for example pushing a button which produces a beeping sound or shaking a box which makes a noise, facilitate infants' imitation (e.g., Devouche, 1998; Hauf, Elsner, \& Aschersleben, 2004). However, to our knowledge, no prior study has investigated the question whether these principles apply to infants' imitation of gestures as well. Other than in the imitation of object-related actions, gestures cannot have physical effects as they are not directed at the physical world. Rather, as social motivation is emphasized in gesture imitation, social effects could be especially important. A study by Masur and Ritz (1984) may be taken as evidence for the influence of social factors on the imitation of gestures. They showed that 10-through 16-month-old infants imitated more familiar and communicative gestures (waving, headshaking, pointing, and open-hand reaching) than comparable hand and arm gestures without communicative significance (e.g., opening and closing the fist, raising an arm). This finding illustrates that infants' imitation is influenced by the social goals of an action or in other words, the social function of the gesture. Therefore, social effects (e.g., interaction with somebody) should have an influence on the imitation rate of communicative gestures, similar to the influence of physical effects on the imitation of object-related actions.

Comparing the imitation of two different kinds of target actions also poses the question whether infants show a consistent imitation ability or if imitation is rather a specific skill, which depends on the domain of the type of action given (objectrelated action vs. gesture). In other words, are there infants who imitate consistently better than others, regardless of the kind of target action encountered? It is widely known that especially children with autism have difficulties in learning by imitation (e.g., DeMyer et al., 1972; Meltzoff \& Gopnik, 1993). This low level of imitation ability is closely linked to the atypical social, cognitive and communicative development of children with autism (Dawson \& Adams, 1984; Smith \& Bryson, 1994). So far, however, no consensus has been reached about the individual imitation abilities of typically developing infants. Studies on infants' verbal imitation have suggested the existence of a general ability to learn from an imitative situation and that therefore, there are infants who are consistently better imitators than others (Bloom, Hood, \& Lightbown, 1974). Other studies have investigated the correlation among imitation performances assessed for different action types (e.g., gestures, 
object-related actions, verbal imitation). For example, Masur (1993) examined the imitation of a list of behaviors, such as vocalizations and motor actions, in a longitudinal study with infants from 10 to 21 months of age. The findings showed inter-individual differences in the number of imitated behaviors, but also individual stability in the imitation performance across different sessions over time. Brownell (1988) additionally reported cross-category relations between various types of motor imitation. However, several other studies have led to opposing results, finding no or only very low cross-action type correlations in imitation performances, suggesting that imitativeness is rather a learned skill for a particular domain (e.g., Rodgon \& Kurdek, 1977; Snow, 1989).

The aim of the present study, then, was to compare 18-month-old infants' imitation of communicative gestures with the imitation of motorically similar object-related actions. Hence, we tested infants' imitation of pairs of actions which had highly similar motor components but different functions; one action in each pair was object-related which emphasizes the cognitive function to learn and the other one was a gesture with a social-communicative function. For example, the action pointing, i.e., stretching out an arm with the index finger, was used in the gesture condition to point at a picture on the wall and in the object-related action condition as ringing a table bell with the stretched out index finger. These similar pairs of actions were designed to control the influence of motor components and to make it possible to interpret the imitation performance in terms of the different kinds of actions.

In addition, since no study so far has directly investigated the influence of effects - an important factor of object-related action imitation - on the imitation of gestures, we added action effects as an experimental variation. As communicative gestures occur in a social context, their effects were defined as social effects. Therefore, gestures presented by a model in the effect condition were followed by a social response from a second model (e.g., the first model points at the wall and the second model looks at the point), while in the no effect condition the second model was also present but did not show any reaction to the gesture shown by the first model. Effects for object-related actions were sound or visual effects, based on former studies (e.g., Devouche, 1998).

Furthermore, we investigated the relations between the imitation rates of these two kinds of actions to test whether there are infants who consistently learn more from imitation situations than others. The imitation of these two action types could be related, since some aspects of early social interaction may be influenced by general, age-related cognitive constraints (Brownell, 1988).

In sum, the present study tested three hypotheses: First, based on the importance of the social function of imitation at the age of 18 months (e.g., Nielsen, 2006; Carpenter, 2006; Killen \& Uzgiris, 1981), we expected infants to show a significant imitation effect for gestures as well as for object-related actions. Second, we expected that action effects would have an influence on the imitation of both object-related actions (e.g., Bellagamba \& Tomasello, 1999; Hauf et al., 2004) and gestures (cf., Masur \& Ritz, 1984), in that infants would imitate more target actions in the effect conditions than in the no effect conditions. Third, a further question was whether a general imitation ability for different types of actions would be found, as mixed results have been reported so far. We tested this as an exploratory hypothesis by analyzing correlations between the imitation rates of object-related actions vs. gestures. Additionally, we aimed to explore if the development of 18-months-old infants' imitation ability relates to the development of other areas such as cognitive abilities, language development and social-emotional behavior.

\section{Method}

\subsection{1. Participants}

Forty-nine healthy, 18-month-old infants ( $M=18$ months 15 days; SD=21 days, 29 girls) from middle class families living in the metropolitan area of a large city, participated in the study. Five additional infants were tested but not included in the final sample due to crying $(n=2)$, lack of cooperation $(n=2)$ and technical problems $(n=1)$. All participating infants were typically developing, with a mean birth weight of $M=3450 \mathrm{~g}(\mathrm{SD}=491.4)$ and no developmental delay in the domains of language, cognition, adaptive behavior and social-emotional behavior, as assessed by the Bayley Scales of Infant and Toddler Development III (Bayley, 2006).

\subsection{Materials}

The test stimuli consisted of five target objects and the related actions and five motorically parallel gestures, chosen in accordance with former studies (e.g., Abravanel et al., 1976; Zmyj et al., 2012). These studies defined socially communicative actions as familiar everyday gestures, which infants most likely had observed or produced before in everyday situations. Target objects, actions and the effects are shown in Table 1.

Additionally, to investigate infants' abilities in different developmental areas, a list of subtests of the Bayley Scales of Infant and Toddler Development III (Bayley, 2006), namely the cognitive scale, the language scale (measuring both receptive and expressive communication), the social-emotional behavior scale and three subtests (communication, social and motor development) of the adaptive behavior scale were conducted. Language and cognitive scales were based on direct interaction with the infant, whereas social-emotional and adaptive behavior scales were conducted as a caregiver report. 
Table 1

Test objects with two kinds of target actions (object-related actions and gestures).

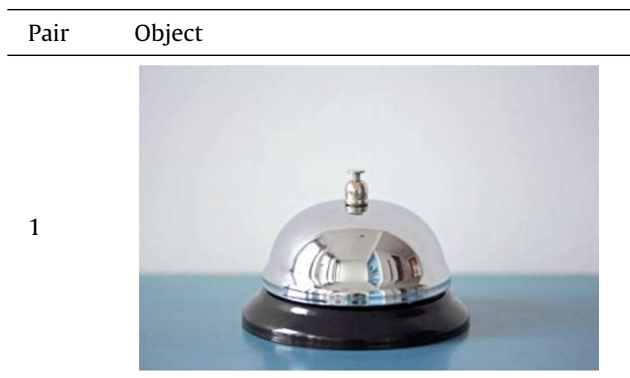

Touching the bell's upper button with the index finger (noise effect/without noise)

Pressing the lamp one arm extended and with the palm up (light effect/without light)

Shaking the tin up and down (noise effect/without noise)

Taking each cymbal between the fingers and bringing them together (noise effect/without noise)

Waving the hand on the surface of the frame (drawing disappears/drawing stays)
"Pointing" (the model extends arm and index finger) (second experimenter looking at the point/no reaction)
"Giving" (the model extends an arm with the palm up toward the second experimenter) (second experimenter giving some cubes in the open hand/no reaction)
"Shaking" (the model shakes one hand down from the wrist) (second experimenter taking the hand and shaking it/no reaction)
"Clapping" (the model claps her hands in front of her body) (second experimenter clapping the hands/no reaction)
"Waving" (the model moves her hand from one side to the other) (second experimenter waving back/no reaction)

Note. Effects of the target actions in the effect condition are printed in italics and no effects of the target actions in the no effect condition are printed in bold.

\subsection{Design and procedure}

Infants were randomly assigned to either the effect group ( $n=17)$ or the no effect group $(n=16)$. To assess the spontaneous production of the object-related target actions in the absence of the demonstration, an additional group of infants was recruited and assigned to a control group $(n=16)$. The parents and the infants were escorted to a reception room, where a warm-up phase was conducted. The purpose and procedure of the study were explained to the caregiver and written 
informed consent was obtained ${ }^{1}$. After this, the caregiver was asked to fill in the Bayley questionnaire of social-emotional and adaptive behavior. Meanwhile, the child could get familiar and play with the two experimenters. Testing began after approximately $10 \mathrm{~min}$ of warm-up play. Experimental sessions took place in a sparsely furnished testing room where the child was seated on the caregiver's lap at a table, opposite to the two experimenters, who were sitting next to each other. Caregivers were asked not to give any hints about what to do with the objects and not to name the gestures and objects.

In the demonstration session of the effect group and the no effect group, each infant participated in ten test trials, that is, five object-related trials and five gesture trials. To control for effects based on temporal order of action presentation, the infants were randomly assigned to two different presentation orders. In the condition "presenting order 1 " $(n=13)$ the demonstrations with object-related actions were presented in the first, third, fifth, seventh and ninth position. The gestures were presented between the object-related actions. In the condition "presenting order 2 " $(n=20)$ the demonstrations with gestures were presented for the first, third, fifth, seventh and ninth positions and the object-related actions were presented between the gestures. In addition, within the two presentation orders, target actions (both object-related actions and gestures) were presented in a randomized order. The infant and the model were videotaped by two cameras. After the experimental session of the imitation study, different subtests of the Bayley Scales were conducted.

\subsubsection{Demonstration of object-related actions}

After a warm-up play with a toy, the demonstration phase began. The second experimenter retrieved the first object from a hidden container below the table and placed it on the table in front of the child. Therefore, the second experimenter was also involved in the procedure to ensure similar activity levels of the two experimenters. Then, the first experimenter started to demonstrate the target action, saying: "Look, [Name], I will show you something!" The model demonstrated the target action two times within roughly $30 \mathrm{~s}$. Then, the object was handed over to the infant while the model said: "Now it's your turn". The infant was given $30 \mathrm{~s}$ to play with the object starting from the moment when the model removed her hands from the object. The same procedure was repeated for all target objects. The target actions were either followed by an effect (effect group) or no effect (no effect group).

\subsubsection{Baseline assessment for the object-related actions}

A baseline control for the object-related actions was conducted with an independent control group $(n=16)$. The experimenter placed the first object in front of the infant and directed the infant's attention to it, saying: "Look, [Name], you can play with this." After 30 s, the experimenter removed the object and put the next object on the table, saying: "Look, [Name], now you can play with this one". The same procedure was repeated for all target objects, presented in a randomized order.

\subsubsection{Demonstration of gestures}

In the gesture demonstration phase the first experimenter started the demonstration by looking at the infant and saying: “Look, [Name], I will show you something!" Then the first experimenter turned to the second experimenter, who was sitting close by and facing the first experimenter, and demonstrated the target gestures two times within roughly $30 \mathrm{~s}$. In the effect condition, the second experimenter showed a social response. In the no effect condition, the second experimenter showed no response. After the demonstration, the first experimenter turned to the infant saying: "Now it's your turn". The infant was given $30 \mathrm{~s}$ to imitate the gesture. The same procedure was repeated for all gestures.

\subsubsection{Baseline assessment for the gestures}

Baseline performance of gestures was analyzed from the video by a trained observer following the coding scheme in previous studies (Zmyj et al., 2012; Jones, 2007). Each demonstration phase of the former gesture served as a baseline for the spontaneous production of the next gesture. Therefore, for example, it was analyzed whether the infant had produced the second gesture, pointing, during the modeling of the first gesture, waving. Similarly, the demonstration phase of the second presented gesture, pointing, was used as a baseline for the third presented gesture. The demonstration phase of the third presented gesture was used as a baseline for the fourth gesture and the fourth demonstration phase was used as a baseline for the last presented gesture. The last demonstration phase was used as a baseline for the first presented gesture.

\subsection{Coding procedure}

A naive rater scored the performance of the target actions in the videotaped sessions. In addition, one-third of the videotapes were scored by a second rater. A good inter-rater reliability was obtained, $k=.83(p<.001)$. Particularly, the reliability for object-related imitation was $k=.92(p<.001)$ and for gestures $k=.73(p<.001)$. For each task, infants received a score of 1 when they produced the target action and otherwise a score of 0 . Therefore, infants could receive an imitation score from 0 to 5 for each action type (object-related actions versus gestures). A third naïve rater used a stopwatch to measure the object manipulation time and the overall attention time (proportion of looking time during the observation of the demonstration phase) for the object-related actions and gestures in the videotaped sessions.

\footnotetext{
${ }^{1}$ The study has been conducted in full accordance with the ethical guidelines of the German Psychological Society and is also in line with the Ethical Principles of Psychologists and Code of Conduct of the American Psychological Association.
} 


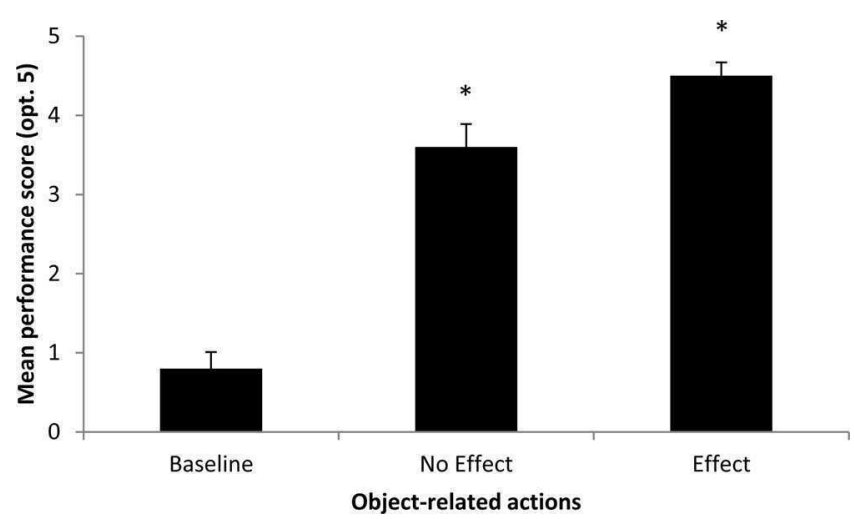

Fig. 1. Mean performance scores of object-related actions in the baseline and the imitation conditions. Error bars indicate standard errors and asterisks indicate significant differences relative to baseline performance.

\section{Results}

\subsection{Preliminary analyses}

As data were not distributed normally, nonparametric tests were employed. Preliminary analyses showed that the presentation order (presenting order 1 vs. presenting order 2 ) did not affect imitation rate, $U=120.50, z=-.35, p=.723, r=-.06$. This factor was therefore not included in any of the following analyses. Furthermore, Mann-Whitney $U$ tests were conducted to test whether the between-subject factor effect (effect versus no effect) led to a difference in infants' interest or attention to the actions. No main effect of the factor effect was found regarding object manipulation time $(U=127.00, z=-.32, p=.746$, $r=-.06)$, overall attention time during the demonstration phases of object-related actions $(U=111.00, z=-.90, p=.357$, $r=-.16)$ and overall attention time during the demonstration phase of gestures $(U=105.00, z=-1.12, p=.264, r=-.19)$.

\subsection{Imitation effect}

To test whether a significant imitation effect for gestures and object-related actions exists, the imitation scores in the baseline vs. in the experimental condition (object-related actions), as well as in the baseline phase vs. in the experimental phase (gestures) were compared. Mann-Whitney $U$ tests revealed that infants in the experimental conditions performed significantly more object-related actions than infants in the control condition (effect condition: $U=.00, z=-5.02, p<.001$, $r=-.87$; no effect condition: $U=8.00, z=-4.60, p<.001, r=-.81$ ), as shown in Fig. 1 . Similarly, a Wilcoxon signed-rank test revealed that infants performed significantly more gestures in the experimental phase than in the baseline phase (effect condition: $z=-3.48, p=.001, r=-.60$; no effect condition: $z=-2.97, p=.003, r=-.53$ ), as shown in Fig. 2.

\subsection{Effects of action type and action effects}

A Wilcoxon signed-rank test was used for within group comparisons (object-related actions versus gestures) to test whether infants' imitation performance varied as a function of action type and a Mann-Whitney $U$ test was used for

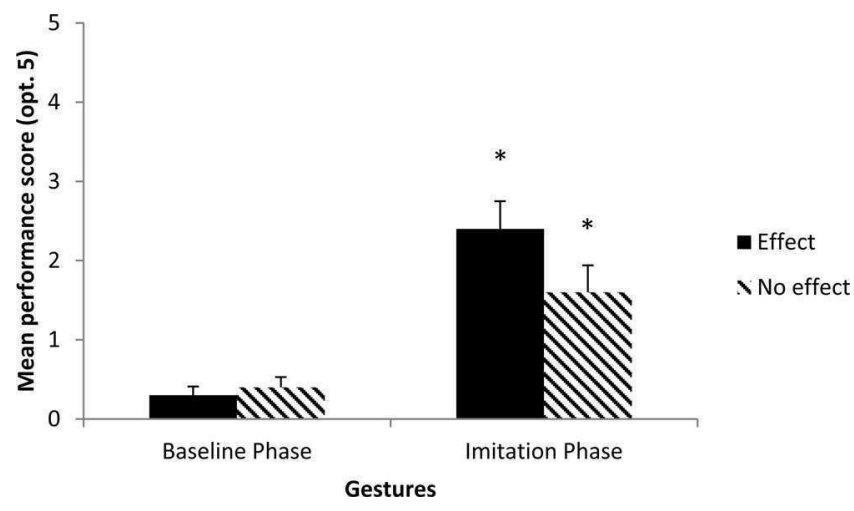

Fig. 2. Mean performance scores of gestures in the baseline and the imitation phase. Error bars indicate standard errors and asterisks indicate significant differences relative to baseline performance. 


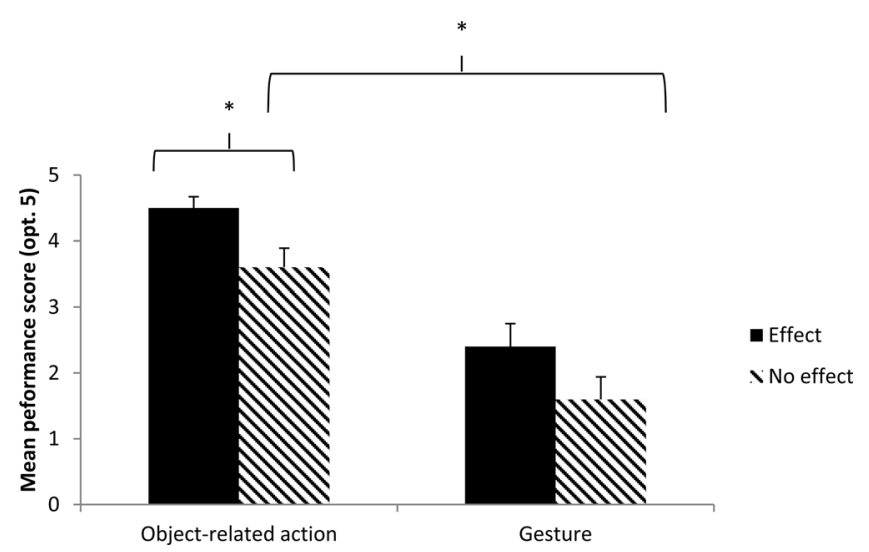

Fig. 3. Mean performance scores for object-related actions vs. gestures in the two effect conditions. Error bars indicate standard errors and asterisks indicate significant differences, $p<.05$.

comparison between the two groups (effect versus no effect) to test whether action effects affected infants' imitation behavior. The analysis revealed that object-related actions were imitated significantly more frequently than gestures $(z=-4.50$, $p<.001, r=-.55)$, regardless if an effect was present $(z=-3.22, p=.001, r=-.55)$ or absent $(z=-3.10, p=.002, r=-.55)$. Further analysis revealed that the imitation rate of object-related actions with effect $(M=4.5, \mathrm{SE}=.17)$ was significantly higher than the imitation rate of the same type of actions without effect $(M=3.6, \mathrm{SE}=.29), U=72.00, z=-2.44, p=.015, r=-.42$. However, infants' imitation rate of gestures followed by an effect $(M=2.4, \mathrm{SE}=.35)$ was not significantly higher than the imitation rate of gestures without effect $(M=1.6, \mathrm{SE}=.34), U=94.00, z=-1.56, p=.119, r=-.27$. The mean imitation rate of object-related actions and gestures in the effect and no effect conditions are shown in Fig. 3.

Additionally, descriptive analysis on item level was conducted to examine whether the imitation rate in the presence of an effect was only found on the overall imitation rate or also on item level. These findings are given in Fig. 4 . The results of object-related actions showed that each object-related action - except for the object 'frame' - was imitated by more infants in the effect condition than in the no effect condition. The results of gestures on item level showed that the gestures pointing and giving were imitated by twice as many infants in the effect condition than in the no effect condition. In contrast to these two gestures, the remaining three gestures shaking, clapping and waving had a similar, low, imitation rate. Furthermore, an item-specific descriptive analysis of the baseline rate revealed that with each object - except for the gesture 'pointing' infants imitated the target actions more frequently in the test phase than in the baseline phase.

\subsection{Imitation as a general ability}

Next, correlations between the imitation rates of the two types of actions were examined. A Spearman's rank correlation test found a significant relation between object-related action imitation and gesture imitation (Spearman's $p=.44, p=.010)$, which shows that infants who imitated more object-related actions also imitated more gestures and vice versa. Furthermore, correlations between infants' imitation abilities and general characteristics as well as test scores in different developmental areas were investigated. No significant correlations were found between imitation rates and gender (Spearman's $p=.14$, $p=.430$ ), age (Spearman's $p=.15, p=.413$ ), cognitive abilities (Spearman's $p=-.27 p=.210$ ), language development (receptive language, Spearman's $p=-.09, p=.680$; expressive language, Spearman's $p=.21, p=.352$ ) and social-emotional behavior (Spearman's $p=.50, p=.795$ ), as measured by the Bayley Scales of Infant and Toddler Development III (Bayley, 2006).

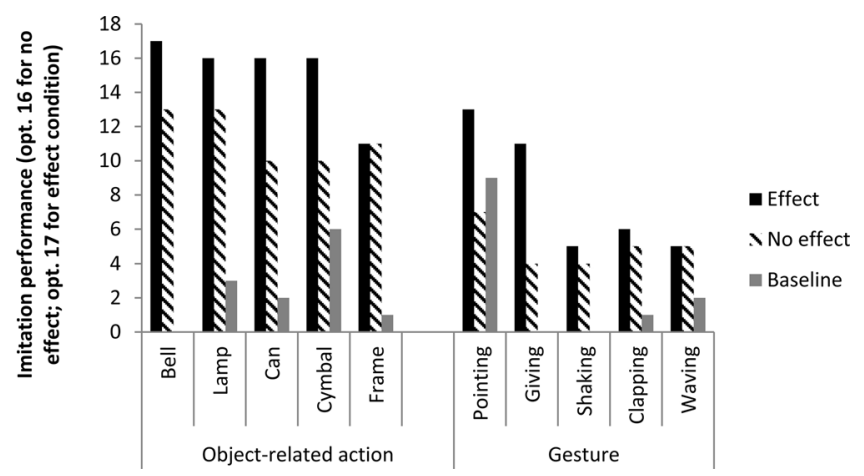

Fig. 4. Baseline as well as imitation rate for the different target actions (object-related actions vs. gestures) in the two effect conditions. 


\section{Discussion}

The present study investigated 18-month-old infants' imitation of gestures compared to object-related actions, as well as the influence of action effects on the imitation rate of these two types of actions. Importantly, the present study used motorically similar pairs of actions to exclude the interpretation of the results based on motor incapacity. The findings showed that 18-month-old infants are less likely to imitate communicative gestures compared to object-related actions, even though they have the motor ability to imitate them. Furthermore, the presence of an effect significantly increased the overall imitation rate of object-related actions; however, social effects did not significantly increase the overall imitation rate of gestures. Finally, we investigated if a general imitation ability for object-related actions and gestures exists. The results demonstrated that infants who imitated one type of actions with greater frequency showed a higher imitation rate of the other type of actions as well, indicating a general imitation ability.

The results confirmed our expectations that 18 -month-olds would imitate object-related actions as well as gestures. This is in line with previous findings which have pointed out that infants from this age onwards are more inclined to imitate the specific actions of a model (e.g., Carpenter, 2006), due to their motivation to maintain the interaction as a sort of imitation game (Uzgiris, 1981). However, infants imitated significantly more object-related actions than non-object-related gestures, despite them having the same motor components. Interpreted in the framework suggested by Uzgiris (1981) and Nielsen (2006), this finding shows that although social motivations start to play a role in imitation at around 18 months of age, infants' imitation is still strongly governed by the cognitive motivation to 'learn', and that learning should be depicted to contextual factors, such as objects. Therefore, the emergent dominance of the social (communicative) function of imitation develops gradually: it becomes more flexible and less context dependent over time, leading to the broadening of action types in which imitation is used.

One additional explanation that needs to be addressed concerns the role of retrieval cues respective to the recall context. When learning about object-related actions, infants can retrieve the information when they see the same or similar objects. The objects themselves serve as retrieval cues, with certain parts or properties pointing to a function that is relevant in effect attainment. Accordingly, object-related actions could be easier to recall with respect to existing retrieval cues. Contrary to this, in the case of gestures, there is nothing in the external context that could be used as cue or grounding for what they should perform. Social partners are in fact too general retrieval cues; their presence in itself could not help infants to retrieve a concrete gesture, only if infants were able to identify the meaning the other person would like to exchange. Thus, gesture imitation could be a more difficult task than object-related action imitation.

Our findings, as predicted, demonstrate that effects enhanced infants' imitation in the case of object-related actions, replicating a series of former studies (e.g., Hauf et al., 2004). However, the prediction that an effect would enhance the overall imitation rate of gestures, as well, was not confirmed. The item-specific analysis of the gestures conducted in the present study demonstrated that only the gestures pointing and giving were imitated more frequently in the effect condition than in the no effect condition. The remaining gestures, waving, clapping and shaking had a similar and low imitation rate in both conditions. Previous studies suggest that in the imitation of gestures aspects of the action or demonstration regarding social influence and socialization might be especially important for imitation. Zmyj et al. (2012), for example, showed that infants were more likely to imitate familiar gestures from a peer model than an older child or adult, in order to identify with the model. The findings in the present study extends prior findings by indicating that infants are more likely to imitate gestures they find familiar and meaningful in the whole context of interaction. The gestures pointing and giving had a meaning in relation to the subsequent effects in the whole context of the demonstration. In the case of pointing, the first experimenter looked at the second experimenter and pointed at a picture on the wall and the second experimenter looked at it. Likewise, giving was demonstrated in the context that the first experimenter implied to give something to the second experimenter and the second experimenter stretched out her hand for it. However, the other gestures waving, clapping and shaking did not occur as a whole interaction situation. For example, the first experimenter started to clap or wave even though nothing had happened before that action. Therefore, it seems that in the imitation of gestures, the aspect of the whole social interaction context is relevant. This interpretation is consistent with findings by Killen and Uzgiris (1981) suggesting that the social meaning of an action pattern rather than familiarity or novelty is an important determinant of imitation.

The current results strongly indicate that different types of gesture imitation must be investigated more thoroughly. Unlike object-related action imitation, infants are not presenting the acquired action "on request" when they imitate gestures. Gesture imitation is rather multifaceted and has many factors and aspects which must be considered when interpreting the findings of imitation studies. Various gestures have various social functions (e.g., getting the attention of somebody else, expression of emotions, greetings); these are distinguished upon their familiarity and if they have a social function by themselves, without a subsequent effect.

A further goal of the study was to investigate if imitation is a learned action type specific strategy or rather a more general ability and accordingly, if infants with consistently low or higher imitation rates exist. The current results show that gesture imitation is significantly related to object-related action imitation; the same infants who were better in imitating gestures were also better in imitating object-related actions. Therefore, the current findings confirm previous data showing that infants' imitation performance is rather a general ability (e.g., Masur, 1993; Brownell, 1988) and extend the results of previous investigations for the general imitativeness of object-related actions and gestures. However, none of the assessed characteristics of infants (i.e., Bayley scores, gender, age) were related to the different imitation abilities. Accordingly, the 
question whether infants' imitative abilities correlate with other facets of social and cognitive development remains open for future investigations.

\section{Conclusions}

To sum up, this study is the first to systematically investigate 18-month-old infants' imitation of object-related actions compared to motorically similar gestures with an experimental variation of physical and social action effects. The current findings suggest that at the age of 18 months, although infants' social motivations are sufficient to evoke the imitation of gestures, imitation rates are still higher in the case of object-related actions. Furthermore, our results show that action effects play an important role for object-related actions; however, an effect did not enhanced imitation rate in all gestures. Rather, infants only showed a higher rate of imitation of gestures with subsequent effects that had a meaning in the whole social interaction context of the demonstration.

\section{Acknowledgements}

We would like to thank the infants and their families who participated in this study. We also want to thank Julia Braun who assisted in conducting the experiment.

\section{References}

Abravanel, E., Levan-Goldschmidt, E., \& Stevenson, M. B. (1976). Action imitation: The early phase of infancy. Child Development: 47., 1032-1044. http://dx.doi.org/10.2307/1128440

Barr, R., \& Hayne, H. (2003). It's not what you know, it's who you know: Older siblings facilitate imitation during infancy. International Journal of Early Years Education: 11., 117-121. http://dx.doi.org/10.1080/0966976032000066055

Bayley, N. (2006). Bayley scales of infant and toddler development (3rd ed.). San Antonio: Harcourt Assessment Inc.

Bekkering, H., Wohlschläger, A., \& Gattis, M. (2000). Imitation of gestures in children is goal-directed. The Quarterly Journal of Experimental Psychology: $53 .$, 153-164. http://dx.doi.org/10.1080/713755872

Bellagamba, F., \& Tomasello, M. (1999). Re-enacting intended acts: Comparing 12- and 18- months-olds. Infant Behavior and Development: $22 ., 277-282$. http://dx.doi.org/10.1016/s0163-6383(99)00002-8

Bloom, L., Hood, L., \& Lightbown, P. (1974). Imitation in language development: If, when, and why. Cognitive Psychology: 6., 380-420. http://dx.doi.org/ 10.1016/0010-0285(74)90018-8

Brownell, C. (1988). Combinatorial skills: Converging developments over the second year. Child Development: 59., 675-685. http://dx.doi.org/10.2307/ 1130567

Carpenter, M. (2006). Instrumental, social, and shared goals and intentions in imitation. In S. J. Rogers, \& J. H. Williams (Eds.), Imitation and the social mind (pp. 49-70). New York, London: The Guilford Press.

Christie, T., \& Slaughter, V. (2009). Exploring links between sensorimotor and visuospatial body representations in infancy. Developmental Neuropsychology: 34., 448-460. http://dx.doi.org/10.1080/87565640902964532

Dawson, G., \& Adams, A. (1984). Imitation and social responsiveness in autistic children. Journal of Abnormal Child Psychology: 12., 209-226. http://dx.doi.org/10.1007/bf00910664

DeMyer, M. K., Alpern, G. D., Barton, S., De Myer, W. E., Churchill, D. W., Hingtgen, J. N., et al. (1972). Imitation in autistic early schizophrenic and non-psychotic subnormal children. Journal of Autism and Childhood Schizophrenia: 2., 264-287. http://dx.doi.org/10.1007/bf01537618

Devouche, E. (1998). Imitation across changes in object affordances and social context in 9-month-old infants. Developmental Science: 1., 65-70. http://dx.doi.org/10.1111/1467-7687.00014

Hauf, P., Elsner, B., \& Aschersleben, G. (2004). The role of action effects in infants' action control. Psychological Research: 68., 115-125. http://dx.doi.org/ 10.1007/s00426-003-0149-2

Jones, S. S. (2007). Imitation in infancy: The development of mimicry. Psychological Science: 18., 593-599. http://dx.doi.org/10.1111/j.14679280.2007.01945.x

Killen, M., \& Uzgiris, I. C. (1981). Imitation of actions with objects: The role of social meaning. The Journal of Genetic Psychology: 138., 219-229. http://dx.doi.org/10.1080/00221325.1981.10534136

Masur, E. F. (1993). Transitions in representational ability: Infants' verbal, vocal, and action imitation during the second year. Merrill-Palmer Quarterly: 39. (4), 437-456.

Masur, E. F., \& Ritz, E. G. (1984). Patterns of gestural, vocal, and verbal imitation performance in infancy. Merrill-Palmer Quarterly: 30., 369-392. http://dx.doi.org/10.1016/b978-0-12-504750-0.50041-7

Matheson, H., Moore, C., \& Akhtar, N. (2013). The development of social learning in interactive and observational contexts. Journal of Experimental Child Psychology: 114., 161-172. http://dx.doi.org/10.1016/j.jecp.2012.09.003

Meltzoff, A. N., \& Gopnik, A. (1993). The role of imitation in understanding persons and developing a theory of mind. In S. Baron-Cohen, H. Tager-Flusberg, \& J. D. Cohen (Eds.), Understanding other minds: Perspectives from autism (pp. 335-366). New York: Oxford University Press.

Nielsen, M. (2006). Copying actions and copying outcomes: Social learning through the second year. Developmental Psychology: 42., 555-565. http://dx.doi.org/10.1037/0012-1649.42.3.555

Over, H., \& Carpenter, M. (2012). Putting the social into social learning: Explaining both selectivity and fidelity in children's copying behavior. Journal of Comparative Psychology: 126., 182-192. http://dx.doi.org/10.1037/a0024555

Rodgon, M. M., \& Kurdek, L. A. (1977). Vocal and gestural imitation in 8-, 14-, and 20- month-old children. Journal of Genetic Psychology: 131., 115-123. http://dx.doi.org/10.1080/00221325.1977.10533280

Smith, I. M., \& Bryson, S. E. (1994). Imitation and action in autism: A critical review. Psychological Bulletin: 116., 259-273. http://dx.doi.org/10.1037/ 0033-2909.116.2.259

Snow, C. E. (1989). Imitativeness: A trait or skill? In G. E. Speidel, \& K. E. Nelson (Eds.), The many faces of imitation in language learning (pp. 73-90). New York: Springer-Verlag.

Uzgiris, I. C. (1981). Two functions of imitation during infancy. International Journal of Behavioral Development: 4., 1-12. http://dx.doi.org/10.1177/ 016502548100400101

Zmyj, N., Aschersleben, G., Prinz, W., \& Daum, M. (2012). The peer model advantage in infants' imitation of familiar gestures performed by differently aged models. Frontiers in Psychology: 3., 1-7. http://dx.doi.org/10.3389/fpsyg.2012.00252 